\title{
BIBECHANA
}

ISSN 2091-0762 (Print), 2382-5340 (Online)

Journal homepage: http://nepjol.info/index.php/BIBECHANA

Publisher: Department of Physics, Mahendra Morang A.M. Campus, TU, Biratnagar, Nepal

\section{Distribution of dust properties around carbon rich AGB star: IRAS 04427+4951 using IRIS and AKARI survey}

\author{
Devendra Raj Upadhyay ${ }^{*}$, Trishna Subedi ${ }^{1}$ \\ ${ }^{1}$ Department of Physics, Amrit Campus, Tribhuvan University, Kathmandu, Nepal \\ *Email: devendra.upadhyaya@ac.tu.edu.np
}

Article Information:

Received: March 3, 2021

Accepted: June 8, 2021

Keywords:

Dust color temperature

Inclination angle

Extinction

OI: https://doi.org/10.3126/bibechana v18i2 35394

\begin{abstract}
Interstellar dust plays a vital role in the interstellar medium, and their properties using far-infrared bands analyze nature around asymptotic giant branch stars and other stellar objects. Here, we present physical properties across the cavity region around an AGB star named IRAS 04427+4951 using Sky View Observatory of IRIS, AKARI map, SIMBAD, Aladin v2.5, and Gaia Archive. The average dust color temperature and mass are $23.48 \pm 0.009 \mathrm{~K}, 3.55 \times 10^{27} \mathrm{~kg}\left(1.79 \times 10^{-3} \mathrm{M}_{\odot}\right)$ using IRIS data and $14.89 \pm 0.004 \quad \mathrm{~K}$ and $5.34 \times 10^{28} \mathrm{~kg}$ $\left(2.69 \times 10^{-2} \mathrm{M}_{\odot}\right)$ from AKARI data. The size of isolated cavity-like structure around the AGB stars of $45.67 \mathrm{pc} \times 17.02 \mathrm{pc}$ and $42.25 \mathrm{pc} \times 17.76 \mathrm{pc}$, respectively. The visual extinction is to be in the range of $3.2 \times 10^{-4}$ to $4.3 \times 10^{-}$ ${ }^{4}$ mag obtained using IRIS survey and $4.5 \times 10^{-3}$ to $7.4 \times 10^{-3}$ mag from AKARI survey. The inclination angle is $86.15^{\circ}$ and $86.08^{\circ}$. The results we present gives the dust distributions, their properties and role which is useful for the study of visual extension phenomena and astrochemistry of interstellar medium.
\end{abstract}

This work is licensed under the Creative Commons CC BY-NC License. https://creativecommons.org/licenses/by-nc/4.0/

\section{Introduction}

The interstellar medium plays a vital role that enables structures in the space between the stars in galaxies $[1,2]$. The physics and astrochemistry around low and intermediate massive stars $(0.8$ 8) $\mathrm{M}_{\odot}$ [3] in Milky-way and other galaxies will enhance research up to polyaromatic hydrocarbon, carbon nanotube in space, and more [2]. These stars evolve and become Asymptotic Giant Branch (AGB) stars having approximately luminous intensity $\left(\mathrm{L}_{\text {star }}=10^{4} \quad \mathrm{~L}_{\odot}\right)$, surface temperature
$\left(\mathrm{T}_{\text {eff }}=3000 \mathrm{~K}\right)$, radius $\left(\mathrm{R}_{\text {star }}=1 \mathrm{AU}\right)$, having mass rates $\left(10^{-7}\right.$ to a few number times $\left.10^{-4} \mathrm{M} \odot / \mathrm{yr}\right)[4,5]$. A dusty environment like flux distribution, dust color temperature, Planck function, dust mass, size of the structure and inclination angle of cavity-like structure the Far Infrared loop, KK loop and pulsar candidate investigated by Jha et al. [6, 7] using both improved reprocessing of IRAS(IRIS) and AKARI survey they obtained the nearly inverse relationship between dust mass and temperature. The environment around Planetary nebulae: NGC 1514, NGC 6826, NGC 2899, and Cone like 
asymmetrical dust structure studied by using IRAS $12,25,60$, and 100 can not be visible by the necked eye, but their heating effect can felt.

Here, we have present our work related to cavities regions around AGB stars by comparing two survey: IRIS and AKARI . So it is new work to understand Far Infrared features around AGB stars. This work based on revised catalog of C-rich AGB stars categorized by Suh \& Hong(2017) [16] and it includes a comparative study of dust features.

This work present in four sections first introduction, second methods, third results and discussion, and the fourth one is the conclusion.

\section{Methodology}

We have taken data of AGB stars using A catalog of AGB stars in our Galaxy

(http://web.chungbuk.ac.kr/ kwsuh/agb.html)

presented by Shu et al. [16-19]. His group presented a revised catalog of 3828 O-rich AGB stars and $1168 \mathrm{C}$-rich AGB stars. We have verified data using SIMBAD Astronomical Database - CDS (Strasbourg)

(http://simbad.u-strasbg.fr/simbad/),

Sky View Virtual Observatory

(https://skyview.gsfc.nasa.gov/current/cgi/query.pl),

Infrared Astronomical Satellite, IRIS - Improved Reprocessing of the IRAS Survey, AKARI survey. The distance of the candidate is taken from Gaia Archive

(https://gea.esac.esa.int/archive/).

We carried out search of different cavity like structures around the different AGB stars in IRIS and AKARI map available in the sky view virtual observatory. The steps that we followed during the search are elaborated below:

Step-I: Inspection of the region in $1^{0} \times 1^{0}$ in sky view virtual observatory: The parameters used during sky survey are listed below:

Coordinate: J2000, Projection: Gnomonic

Image size (pixel): $500 \times 500$

Brightness scaling: Histogram Equalization
Step-II: Image taken on the basis of searching isolated cavity structure around AGB star

Step-III: Extraction of flux using Aladin v2.503 software(https://aladin.u-strasbg.fr/aladin.gml): The Flexible Image Transport System (FITS) images were downloaded from sky view, according to the procedure mentioned above and the FITS image is processed using software Aladin v2.503.

\section{Region of interest}

The image structure located at R.A. (J2000) $=04^{\mathrm{hr}}$ $46^{\mathrm{m}} 33.036^{\mathrm{s}}$ and Dec. $(\mathrm{J} 2000)=+49^{0} 56^{\mathrm{m}} 23.77^{\mathrm{s}}$ selected as a region of our interest. The reasons of selections are as follows:

1 The structure seems to be isolated and its size is around $3.24 \mathrm{pc}$ for IRIS and $12.28 \mathrm{pc}$ for AKARI in contraction.

2 There were several condensations (minima) and the features can be seen in all 4 bands of IRIS survey and AKARI survey.

3 The structure in IRIS is prominent in $60 \mu \mathrm{m}$ and $100 \mu \mathrm{m}$ that enable us to study the fluxes emitted from the dust and grain.

4 The structure in AKARI is prominent in $90 \mu \mathrm{m}$ (AKARI WIDE-S) and $140 \mu \mathrm{m}$ (AKARI WIDE-L) that enable us to study the fluxes emitted from the dust and grain.

\section{Data reduction}

Each pixel of the cavity-like region in FITS images are analyzed by using Aladin v2.503 which helps in data reduction. With the help of Aladin v2.503 the invisible spectral photography can be reduced into desired color and analysis of the relative flux distribution in the region of interest can be done. More precisely we cannot make visible pictures and photography in IR region but, using this software we can make visible reference with color specification. So it is convenient to draw contour map, hexbin plot and marking to find desired physical parameter (e.g. Flux, coordinate, etc). The Aladin software can be considered as sky ATLAS designed by CDS for research of astronomical sources through color coded display of required 
image. Aladin v2.503 allows us to visualize digitized images of any part of the sky, to superimpose entries from the CDS astronomical catalogs and tables, and to interactively access related data and information from SIMBAD and Gaia Archive of all known objects in the field.

\section{Flux density variation}

We studied the flux density of all the pixel lying inside the counter level 71 for IRIS under $100 \mu \mathrm{m}$ and find the flux of corresponding pixels in $60 \mu \mathrm{m}$ using the software Aladin v2.503 which was used to calculate the dust color temperature and mass of each pixel due to the contribution of dust. Similarly, for AKARI we used the counter level 100 under $140 \mu \mathrm{m}$ image and the corresponding pixels in $90 \mu \mathrm{m}$ were obtained. Here we found the regions of maximum and minimum flux and studied the density in each image of both IRIS and AKARI in a comparative manner.

\section{Dust color temperature}

The contour map has been made in $100 \mathrm{~m}$ and 140 $m$ FITS. Then the corresponding flux densities are calculated from the IRIS $60 \mu \mathrm{m}$ image. Using the ratio of the flux density in the IRIS $60 \mu \mathrm{m}$ to the flux density in the IRIS $100 \mu \mathrm{m}$ in the expression given below $[20,21]$, dust color temperature is calculated as,

$T_{d}=\frac{-96}{\ln \left(R \times 0.64^{3+\beta}\right)}$

where, $R=\frac{F(60 \mu m)}{F(100 \mu m)}$

Since, $F(60 \mu \mathrm{m})$ and $F(100 \mu \mathrm{m})$ are the flux densities at $60 \mu \mathrm{m}$ and $100 \mu \mathrm{m}$ respectively.

For AKARI survey, following expression is used to calculate the dust color temperature $[6,20,21]$,

$T_{d}=\frac{-57}{\ln \left(R \times 0.64^{3+\beta}\right)}$

where, $R=\frac{F(90 \mu m)}{F(140 \mu m)}$

Since, F $(90 \mu \mathrm{m})$ and $F(140 \mu \mathrm{m})$ are the flux densities at $90 \mu \mathrm{m}$ and $140 \mu \mathrm{m}$ respectively.

\section{Dust mass estimation}

The dust masses are estimated from the far-infrared flux densities at $100 \mu \mathrm{m}$ image in IRIS and 140 $\mu \mathrm{m}$ in AKARI survey. The infrared flux can be measured from the IRIS sky view images and images from Groningen using Aladin v2.503. The resulting dust mass depends on the physical and chemical properties of the dust grains, the adopted dust temperature and the distance to the object. The final expression for the dust mass [22-24], can be written as;

$M_{\text {dust }}=\frac{4 \mathrm{a} \rho}{3 Q_{v}}\left(\frac{F_{v} D^{2}}{B(v, T)}\right)$

Here,

$\mathrm{B}(v, \mathrm{~T})=$ Planck's function, which is the function of the temperature and frequency, and given by the expression;

$B(v, T)=\frac{2 h v^{3}}{c^{2}}\left(\frac{1}{e^{\frac{h v}{\mathrm{KT}}}-1}\right)$

and

$\mathrm{a}=$ Weighted grain size $=0.1 \mu \mathrm{m}$

$\rho=$ Grain density $=3000 \mathrm{kgm}^{-3}$

$\mathrm{Q}_{v}=$ grain emissivity $=0.0010$ for $100 \mu \mathrm{m}$ and 0.0046 for $60 \mu \mathrm{m}$ respectively.

$\mathrm{F}_{\mathrm{v}}=$ total flux density of the region whose mass is to be determined,

$F_{v}=f \times 5.288 \times 10^{-9} \mathrm{MJysr}^{-1}$

$1 \mathrm{MJysr}^{-1}=1.26 \times 10^{-19} \mathrm{~kg} . \mathrm{s}^{-2}$

$\mathrm{D}=$ distance to the structure

$\mathrm{B}(v, \mathrm{~T})=$ Planck's function.

$\mathrm{h}=$ Planck's constant

$\mathrm{c}=$ velocity of light

$v=$ frequency at which the emission is observed and $\mathrm{T}=$ the average temperatures of the region. Values of different parameters we use in the calculation of the dust mass in our interest are as follows:

Using these values, an expression (3) takes the form [22-24],

$M_{\text {dust }}=0.40\left(\frac{F_{v} D^{2}}{B(v, T)}\right)$

\section{Visual extinction}

For visual extinction we need optical depth[25]

$\tau_{100}=\left(\frac{F_{100 \mu m}}{B(v, T)_{100}}\right)$

Here $\mathrm{F}_{100 \mu \mathrm{m}}$ in $\mathrm{kgs}^{-2}$ and $\mathrm{B}(v, \mathrm{~T})_{100}$ is corresponding intensity of radiation which is observed at $100 \mu \mathrm{m}$ 
flux in IRIS as proposed by Wood et al. [14]. For visual extinction:

$A_{v}=15.078\left(1-e^{\frac{-\tau_{100}}{641.3}}\right)$

We have uses this formula for AKARI by calculating optical depth for $\tau_{140 \text {. }}$

For AKARI survey, following expression is used to calculate the visual extinction

$A_{v}=15.078\left(1-e^{\frac{-\tau_{140}}{641.3}}\right)$

where $\tau_{140}=\left(\frac{F_{140 \mu m}}{B(v, T)_{140}}\right)$

\section{Inclination angle}

The angle between line-of-sight and the plane of celestial object projected in the sphere is called inclination angle. Holmberg (1946) [26] established a simple model to find the inclination angle of celestial objects. Holmberg equation is the relation between the axial ratio ( $b / a)$ and the inclination angle (i), the angle between the line-of-sight and normal to the galactic plane. The flatness factor (q) $=0.33$ is assumed by considering oblate spheroid.

$$
\cos ^{2} i=\frac{\frac{b^{2}}{a^{2}}-q^{2}}{q^{2}}
$$

is called 'Holmberg Formula' for inclination angle [26].

\section{Results and Discussion}

In our work, the dust color temperature, intensity of radiation, dust mass and extension are measured using FITS images of $60 \mu \mathrm{m}, 100 \mu \mathrm{m}$ and $90 \mu \mathrm{m}$, $140 \mu \mathrm{m}$.

\section{Projection map of coordinates in R. A. and DECL}

Fig. 1 is the projection map of the right accession and declination. It show larger region of interest in Fig. 1(a) IRIS in comparison with Fig. 1(b) because we have selected image size $1^{0} \times 1^{0}$ for IRIS and $0.5^{0} \times 0.5^{0}$ in AKARI. But we obtained more pixels in AKARI because of high resolution of survey. This projection is lambert projection in nature.
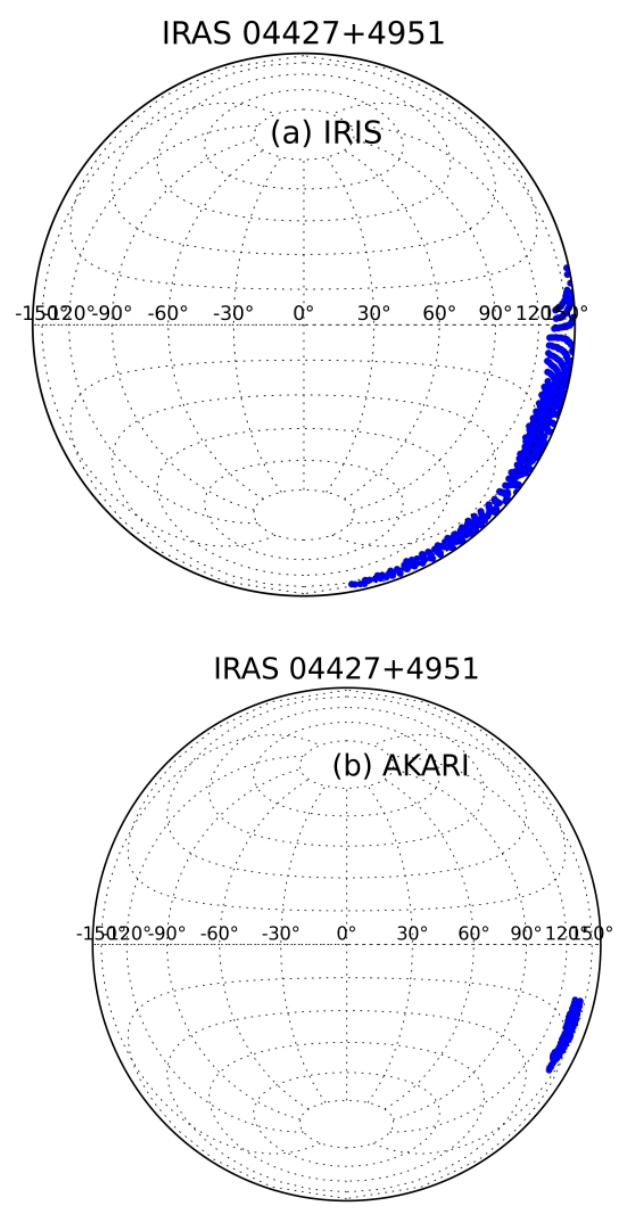

Fig. 1: Position of candidate IRAS $04427+4951$ (a) Projection of coordinates of far infrared cavities using IRIS survey (b) Projection that of coordinates using data of AKARI survey.

\section{Hexbin distributions of flux}

Fig. 2 (a) show the 60 micrometer IRIS image flux distribution in $\mathrm{MJysr}^{-1}$ with R.A.(J2000) and DECL.(J2000) in degree in IRIS survey. Similarly, Fig. 2 (b) show the 90 micrometer AKARI FITS image flux distribution in $\mathrm{MJysr}^{-1}$ with R.A.(J2000) and DECL.(J2000) in degree in IRIS survey. Here, color ranging from violet to red indicates more emission of flux from that pixels. In both case the central region contain lesser flux in low wavelength band of both survey. 

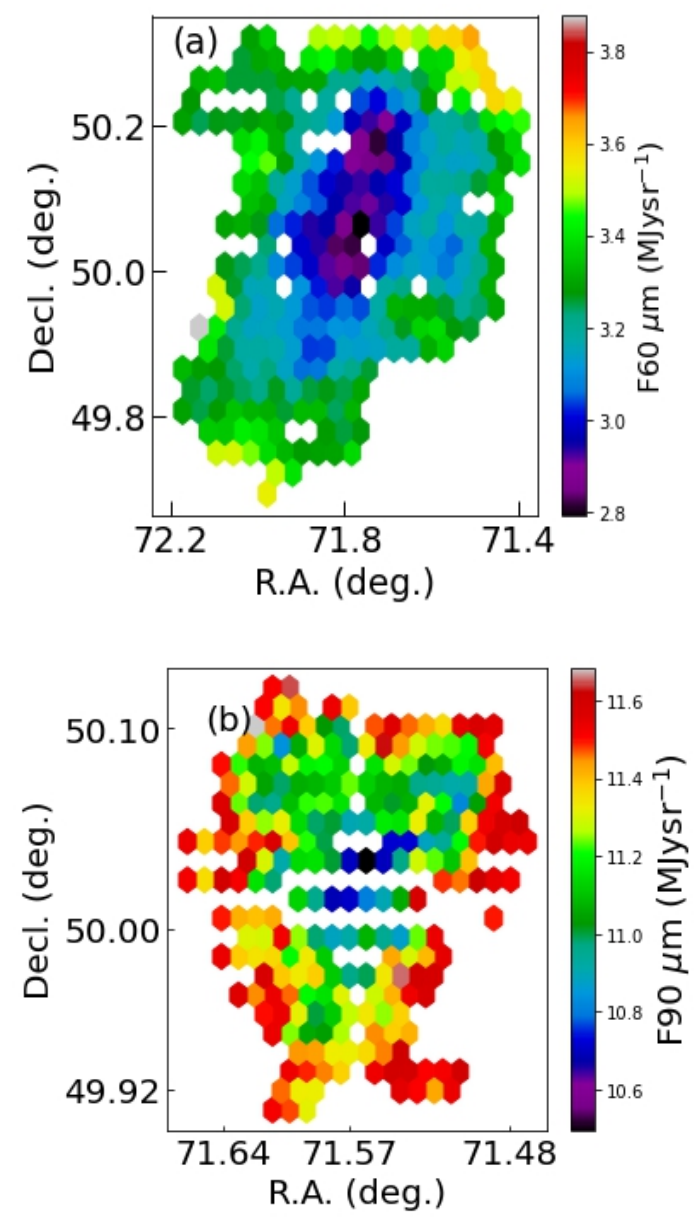

Fig. 2: (a) represents hexbin plot of right ascension (R.A.), declination (DECL.) and $60 \mathrm{~m}$ flux using IRIS survey (b) hexbin plot of right ascension (R.A.), declination (DECL.) and $90 \mathrm{~m}$ flux of IRAS 04427+4951 using AKARI survey.

\section{Mapping of flux}

Fig. 3 (a) present the contour map of 100 micrometer IRIS FITS image flux distribution in MJysr $^{-1}$ with R.A.(J2000) and DECL. (J2000) in degree in IRIS survey. Similarly, Fig. 3(b) show the 140 micrometer AKARI FITS image flux distribution in MJysr-1 with R.A.(J2000) and DECL.(J2000) in degree. Here, color bar ranging from red to violet indicate decrease in flux which show the cavity region of asymptotic giant branch at center in both survey.
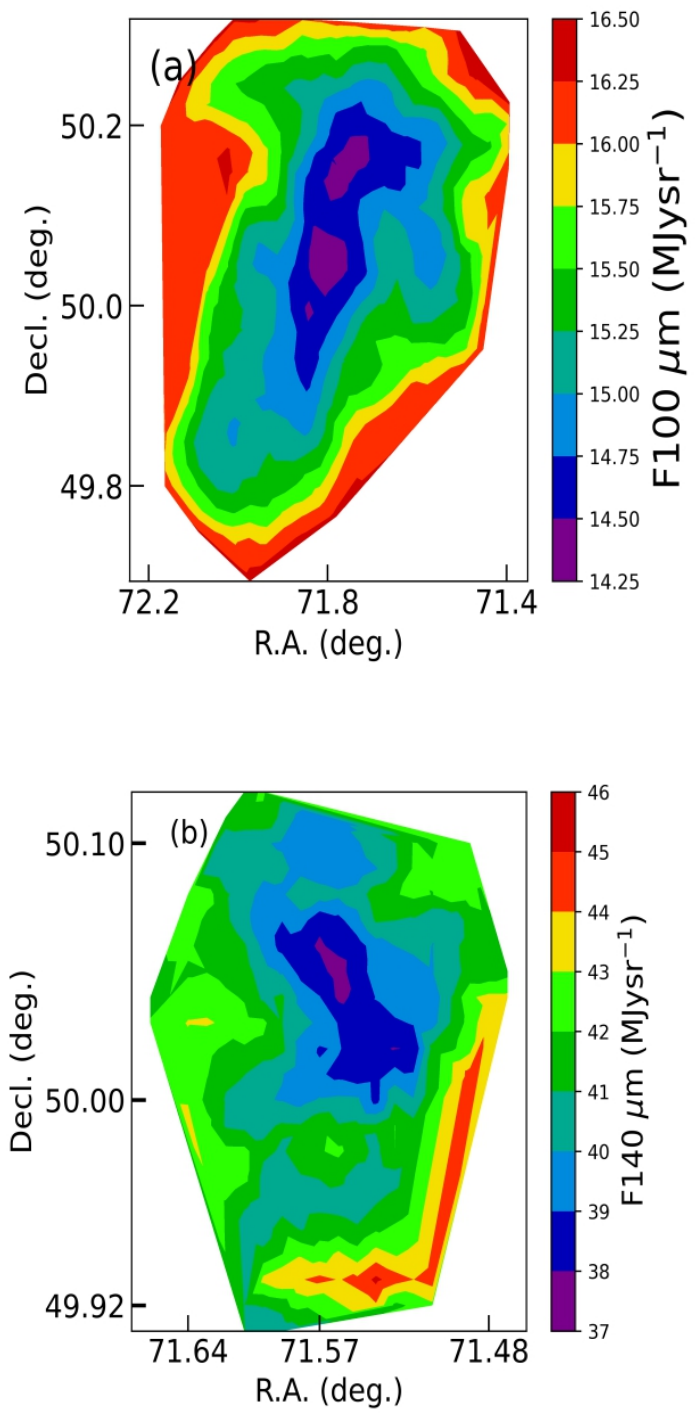

Fig. 3: The contours are shown. (a) $100 \mu \mathrm{m}$ flux (b) $140 \mu \mathrm{m}$ flux with right ascension (R.A.), declination (DECL.) using AKARI survey.

\section{Linear fit of Flux}

Fig. 4(a) represents the best fit between the farinfrared flux of $60 \mu \mathrm{m}$ and $100 \mu \mathrm{m}$. Similarly, Fig. 4(b) shows the best fit relation between far-infrared flux emission in $90 \mu \mathrm{m}$ and $140 \mu \mathrm{m}$. Here, the data extracted from the FITS image of the IRIS and AKARI survey. 

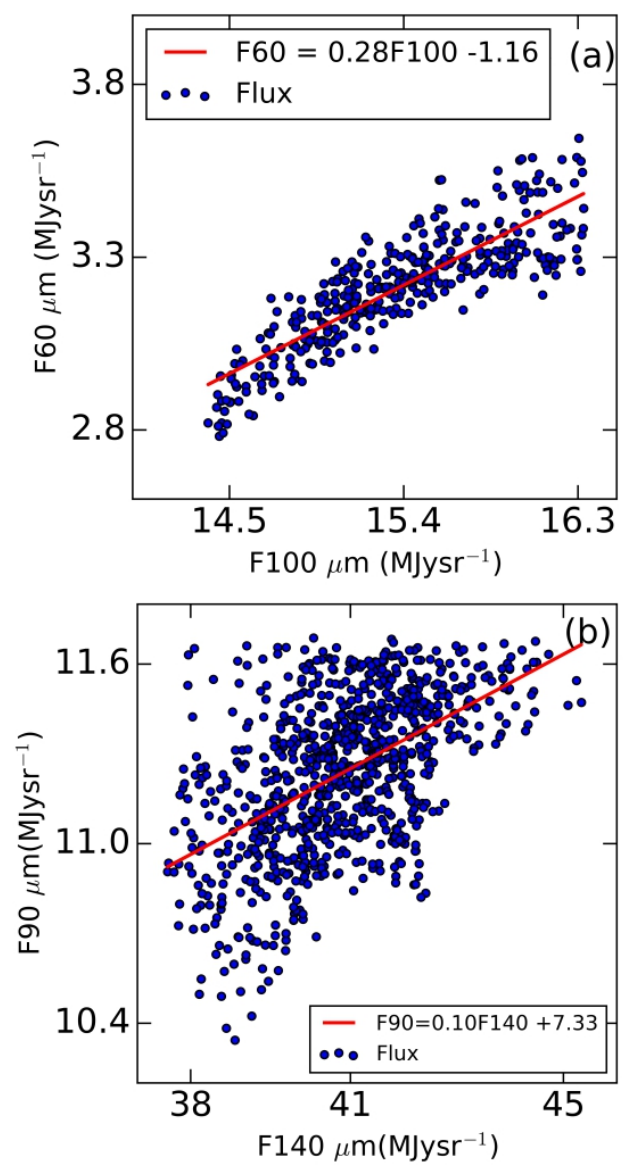

Fig. 4: (a) Linear fit between flux of $60 \mu \mathrm{m}$ and $100 \mu \mathrm{m}$ (b) Linear fit between flux $90 \mu \mathrm{m}$ and 140 $\mu \mathrm{m}$. Both has positive slope.

The best fit line obtained are:

$\mathrm{F} 60=0.28 \mathrm{~F} 100-1.16$

$\mathrm{F} 90=0.10 \mathrm{~F} 140+7.33$

Here, F60, F100, F90 and F140 are flux in $\mathrm{MJysr}^{-1}$. The slope of these line are 0.28 and 0.10 used for the calculation of dust color temperature. The coefficient of correlation 0.85 in IRIS data and 0.53 in AKARI data which shows flux at central region of infrared cavity are more diverse.

\section{Mapping of dust color temperature}

Fig. 5 presents the contour map of dust color temperature distribution. Here, color means the temperature depends upon the wavelength of radiation emitted by a cloud of dust from the cavity region around AGB stars.
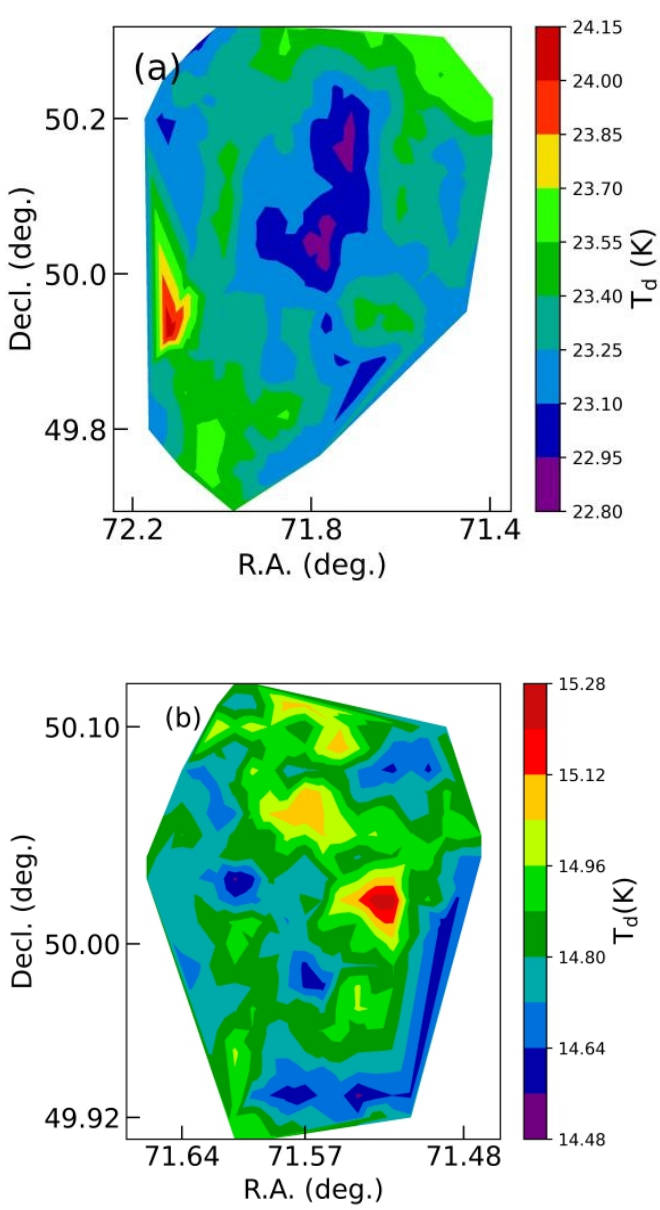

Fig. 5: represents contour map of dust color temperature (a) using IRIS data. (b) using AKARI survey.

The contour and color bar distinguish the color temperature of radiation. On comparing two survey data, the dust color temperature is more in IRIS whereas lesser in AKARI. It shows higher the wavelength band lesser the temperature. From this study, the dust color temperature is in the range between $22.85 \mathrm{~K}$ and $24.1 \mathrm{~K}$ with an average dust color temperature of $23.48 \mathrm{~K}$ in the IRIS survey and $14.54 \mathrm{~K}$ to $15.24 \mathrm{~K}$ with an average dust color temperature of $14.89 \mathrm{~K}$ in the AKARI survey.

\section{Mapping of Planck's function}

Fig. 6 shows intensity of radiation distributions in far-infrared radiation emitted by a cloud of dust around AGB stars corresponding to dust color temperature. 

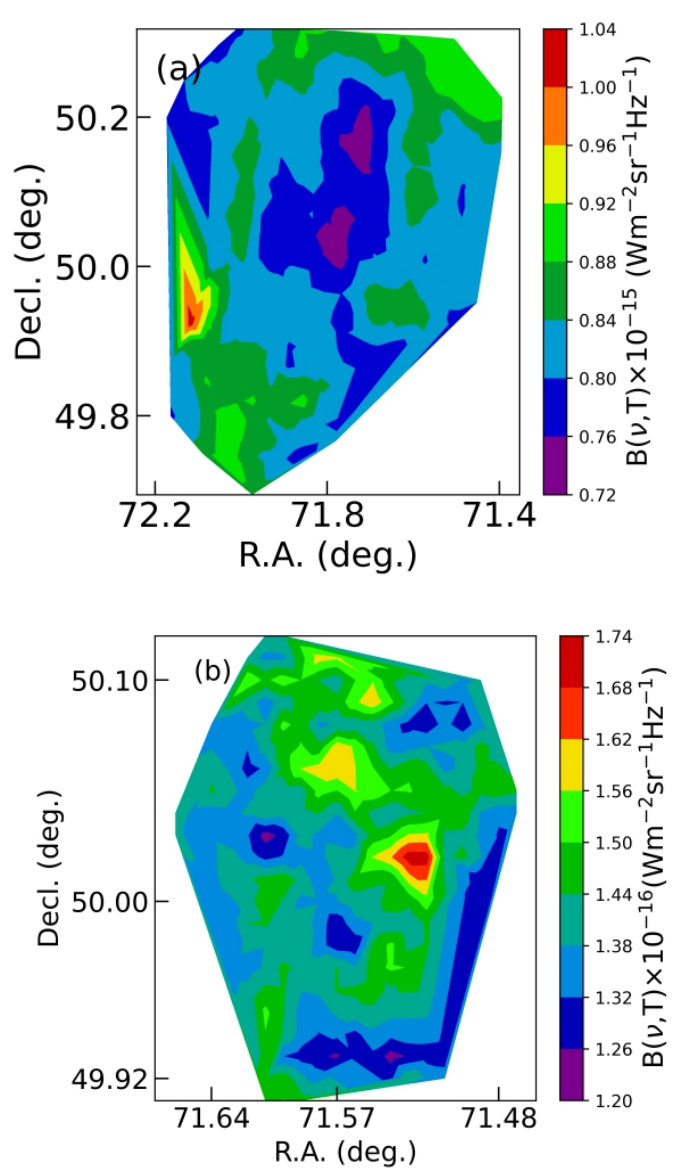

Fig. 6: Contour map of Planck's function (a) using IRIS data. (b) using AKARI survey.

The contours and color separate the different intensities of radiation. In the given Fig. 6(a) and (b), the color bar represents the radiation intensity increases from violet to red. Looking at the isocontours we generated, the IRIS plot shows the spectral intensity decrement towards the center, but the maximum intensity lies a little away from the center. However, the AKARI plot shows the irregular trends in Planck's function values. Here, different in trend due to polytropy nature of the dust in the region around AGB stars.

\section{Mapping of dust mass}

Fig. 7 present the contour map of dust mass in the cavity created due to AGB wind. By calculation we obtained the average dust mass is $3.55 \times 10^{27} \mathrm{~kg}$ $\left(1.79 \times 10^{-3} \mathrm{M}_{\odot}\right)$ using IRIS FITS observation and
$5.34 \times 10^{28} \mathrm{~kg}\left(2.69 \times 10^{-2} \mathrm{M}_{\odot}\right)$ in AKARI FITS analysis. Here on comparing from Fig. 5, Fig. 6, and Fig. 7 in both survey dust mass is more in that region where dust color temperature and Planck's function minimum. It shows a nearly inverse trend of dust mass with the dust color temperature, Planck's function.
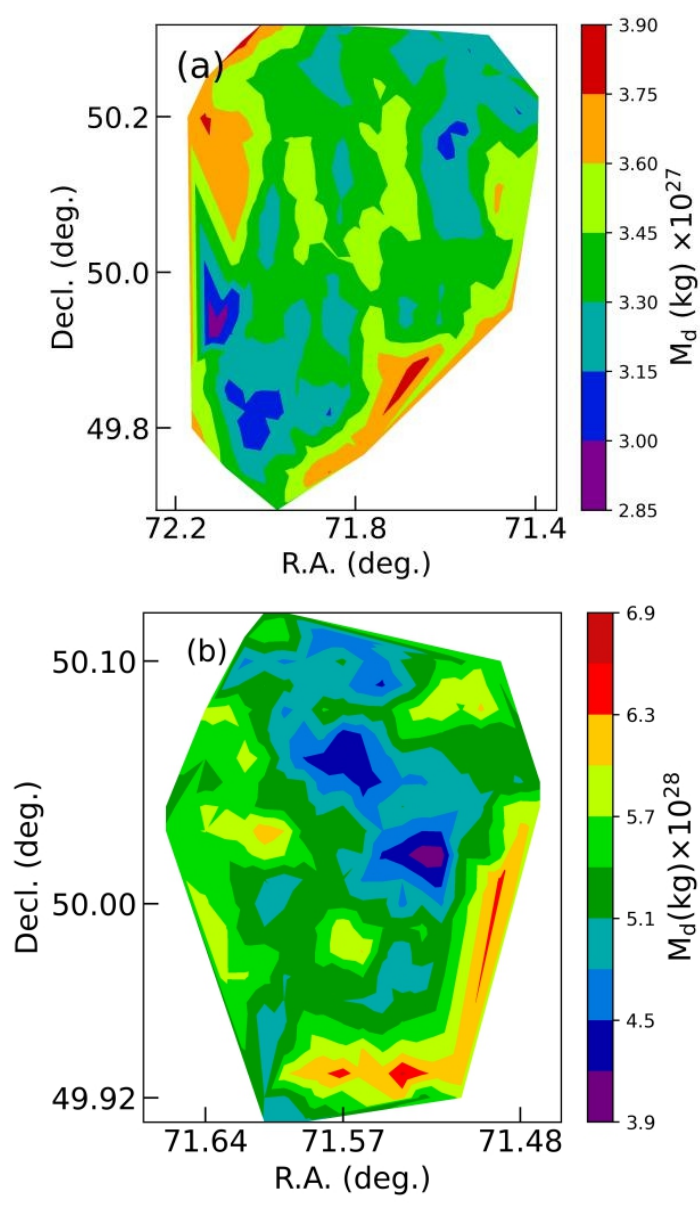

Fig. 7: Contour map of dust mass using ( a) IRIS data. (b) contour map using AKARI survey.

\section{Visual Extinction}

Fig. 8 (a) and (b) show the variation of dust color temperature with visual extinction in both surveys. The values are in the range of $4.3 \times 10^{-4}-3.4 \times 10^{-4}$ mag in IRIS and $7.5 \times 10^{-3}-4.5 \times 10^{-3} \mathrm{mag}$ in AKARI. Here, temperature has inverse relation with visual extinction in both survey. The best fit line for IRIS and AKARI data obtained are: 
$\mathrm{T}_{\mathrm{d}}=-0.75 \mathrm{~A}_{\mathrm{v}}+26.12$

$\mathrm{T}_{\mathrm{d}}=-0.23 \mathrm{Av}+16.16$

The coefficient of correlation are -0.72 in IRIS data and -0.96 in AKARI data. This show that AKARI survey is more intensive and more responsive.
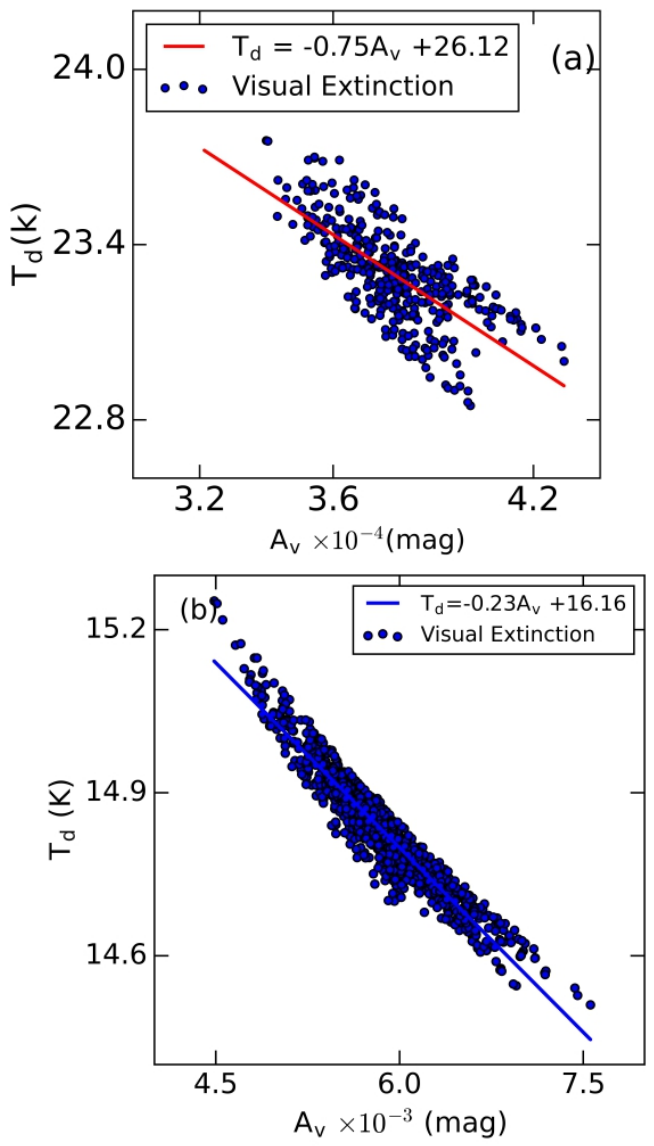

Fig. 8: The variation of dust color temperature $\left(T_{d}\right)$ with visual extinction ( $\left.A_{V}\right)$ (a) for IRIS and (b) for AKARI survey.

Table 1: Structure of the cavity in terms of extension contraction and inclination angle.

Table 1 shows the extension (major diameter)

\begin{tabular}{|c|c|c|c|}
\hline Source & Extension & Contraction & $\begin{array}{c}\text { Inclination } \\
\text { angle }\end{array}$ \\
\hline IRIS & 36.56 & 12.28 & $86.15^{0}$ \\
\hline AKARI & 10.02 & 3.24 & $86.08^{0}$ \\
\hline
\end{tabular}

contraction(minor diameter), inclination angle values. Here, the size of the cavity region we have selected in IRIS is more $(36.56 \times 12.28)$ pc whereas structure is small in AKARI image $(10.02 \times 3.24) \mathrm{pc}$ since we have taken $1^{0} \times 1^{0}$ size in IRIS and $0.5^{0} \times$ $0.5^{\circ}$. Extension and contraction of the structure obtained using a simple expression $\mathrm{L}=\mathrm{R} \times \theta$, where $\mathrm{R}=3000.36 \mathrm{pc}$ is the distance to the structure and $\theta=$ pixel length (in radian). The value of inclination angle is nearly $90^{\circ}$ shows cavity structure suggesting an edge-on appearance.

\section{Conclusions}

We have studied physical properties (flux variation, dust color temperature, dust mass estimation, distance, Planck's function, and extinction) of the cavity region around structure IRAS $04427+4951$ within $1^{0} \times 1^{0}$ for IRIS all-sky map and $0.5^{0} \times 0.5^{0}$ for AKARI all-sky map. We focus on the dust environment of AGB stars. The search of the dust structure in far-infrared $(100 \mu \mathrm{m}$ and $60 \mu \mathrm{m})$ of various AGB stars in IRIS database and $(140 \mu \mathrm{m}$ and $90 \mu \mathrm{m})$ in AKARI all-sky map perform. The distance to the AGB star is $3000.36 \mathrm{pc}$. The size of cavity-like structure around AGB star in IRIS all sky map is found to be $36.56 \mathrm{pc} \times 12.28 \mathrm{pc}$ and AKARI all sky map is found to be $10.02 \mathrm{pc} \times 3.24$ pc. The difference in the obtained size is found due to different size during target extraction i.e., $1^{0} \times 1^{0}$ for IRIS all sky map and $0.5^{\circ} \times 0.5^{\circ}$ for AKARI all sky map and also due to the independent contour map drawn for different map. From the IRIS and AKARI map, we have seen the minimum flux region is at the center of the cavity. The Physical properties of a cavity of our region of interest are studied.

We have extensively used the help of Aladin v2.5 and Python 3. We tagged each pixel and the data of flux density extracted and dust color temperature, dust mass estimation, size of the structure, inclination angle, and visual extinction. From the above study, we obtain the dust color temperature in the range between $22.85 \mathrm{~K}$ and $24.1 \mathrm{~K}$ with an average dust color temperature of $23.48 \mathrm{~K}$ in the 
IRIS survey and $14.54 \mathrm{~K}-15.24 \mathrm{~K}$ with an average dust color temperature of $14.89 \mathrm{~K}$ in the AKARI survey. The average dust mass is $3.55 \times 10^{27} \mathrm{~kg}$ $\left(1.79 \times 10^{-3} \mathrm{M}_{\odot}\right)$ in IRIS survey and $5.34 \times 10^{28} \mathrm{~kg}$ $\left(2.69 \times 10^{-2} \mathrm{M}_{\odot}\right)$ in AKARI survey.

The dust color temperature and dust mass show a nearly inverse relation with each other which supports our physics.

The average value of spectral density is $8.75 \times 10^{-16}$ $\mathrm{Wm}^{-2} \mathrm{sr}^{-1} \mathrm{~Hz}^{-1}$ and $1.4710^{-16} \mathrm{Wm}^{-2} \mathrm{sr}^{-1} \mathrm{~Hz}^{-1}$ using FITS of IRIS and AKARI

The size of the structure is found to be $36.56 \mathrm{pc} \times$ $12.28 \mathrm{pc}$ with inclination angle $86.15^{\circ}$ in IRIS and $10.02 \mathrm{pc} \times 3.24 \mathrm{pc}$ with inclination angle $86.08^{\circ}$ in AKARI map. The visual extinction is found to be in the range of $4.3 \times 10^{-4}-3.4 \times 10^{-4} \mathrm{mag}$ in IRIS and $7.5 \times 10^{-3}-4.5 \times 10^{-3} \mathrm{mag}$ in AKARI. On comparing past dust properties obtain using IRIS and AKARI and our results.

Here, we reach an understanding of lesser dust color temperature, more mass, and extension. It supports the structure such as polyaromatic hydrocarbon, graphene, etc. formation process in the interstellar medium. The dust properties around C-rich, O-rich, S-rich, and Si-rich will do using the spitzer, 2Mass, and WISE survey.

\section{Acknowledgments}

The referee's constructive critique and valuable suggestions are much appreciated. We acknowledge SIMBAD Astronomical Database CDS (Strasbourg), Sky View Virtual Observatory, Infrared Astronomical Satellite, IRIS - Improved Reprocessing of the IRAS Survey, Gaia Archive, and a catalog of AGB stars in our Galaxy (Version 2017) for data. This work under Innovation SRDI75/76 S T 11 supported by University Grants Commission, Nepal.

\section{References}

[1] A. Li, Spitzer's perspective of polycyclic aromatic hydrocarbons in galaxies, Nat. Astron. 4 (2020) 339-351. https://doi.org/10.1038/s41550-020-1051-1
[2] B. T. Draine, Interstellar dust grains, Annu. Rev. Astron. Astrophys. $41 \quad$ (2003) 241-289. https://doi.org/10.1146/annurev.astro.41.011802.0948 $\underline{40}$

[3] B. Aryal et al., A giant dusty bipolar structure around the planetary nebula NGC 1514, Mon. Not. R. Astron. Soc. 402 (2010) 1307-1312. https://doi.org/10.1111/j.1365-2966.2009.15966.x

[4] M. P. Redman et al., A $500 \mathrm{~km} \mathrm{~s}^{-1}$ outflow from the young bipolar planetary nebula $\mathrm{Mz} 3$, Mon. Not. R. Astron. Soc. 312 (2000) L23-L27. https://doi.org/10.1046/j.1365-8711.2000.03327.x

[5] I. Iben, A. Renzini, Asymptotic giant branch evolution and beyond, Annu. Rev. Astron. Astrophys. 21 (1983) 271-342. http://adsabs.harvard.edu/full/1983ARA\%26A..21..27 1I

[6] A. K. Jha, B. Aryal. Dust color temperature distribution of two FIR cavities at IRIS and AKARI maps, J. Astrophys. Astron. 39 (2018) 1-7. https://doi.org/10.1007/s12036-018-9517-6

[7] A. K. Jha et al., A study of dust color temperature and dust mass distributions of four far infrared loops, Rev. Mex. Astron. Astrofis. 53 (2017). http://www.scielo.org.mx/scielo.php?pid=S0185$11012017000200019 \&$ script $=$ sci arttext

[8] B. Aryal et al., Planetary nebulae NGC 6826 and NGC 2899: early aspherical mass loss?, Astrophys. Space Sci. 323 (2009) 323-327. https://doi.org/10.1007/s10509-009-0076-9

[9] B. Aryal, R. Weinberger, A new large high latitude cone-like far-IR nebula, A \& A. 448 (2006) 213219.

https://doi.org/10.1051/0004-6361:20042440

[10] D. R. Upadhyay et al., Dust Structure Around Asymptotic Giant Branch Stars, Proc Int Astron Union. 14 (2018) 525-526. https://doi.org/10.1017/S1743921318007858

[11] A. K. Jha, D. R. Upadhyay, Dust Structure around two Asymptotic Giant Stars at Latitude $32^{\circ} \&$ 40.67 , Himalayan Physics (2017) 41-47. https://doi.org/10.3126/hj.v6i0.18356

[12] L. Khanal et al., Study of ambient environment around Asymptotic Giant Branch Carbon Star: IRAS 01142+ 6306, BIBECHANA 16 (2019) 3140.

https://doi.org/10.3126/bibechana.v16i0.20998

[13] A. K. Gautam, B. Aryal, A study of four lowlatitude $\left(1<10^{\circ}\right)$ far-infrared cavities, J. Astrophys. Astron. 40 (2019) 1-10. 
https://doi.org/10.1007/s12036-019-9578-1

[14] A. K. Gautam, B. Aryal. Study of dust color temperature and visual extinction distribution of a far infrared cavity at 60 and $100 \mu \mathrm{m}$ IRAS map around the AGB star at galactic latitude $8.6^{\circ}$, BIBECHANA 17 (2020) 42-49. https://doi.org/10.3126/bibechana.v17i0.25839

[15] D. O. Wood et al., IRAS images of nearby dark clouds, Astrophys. J. Suppl. Ser. 95 (1994) 457501.

https://doi.org/10.1086/192107

[16] K. W. Suh,Y. J. Kwon, A Catalog of AGB Stars in IRAS psc. J. Korean Astron. Soc. 42 (2009) 81-91. https://doi.org/10.5303/JKAS.2009.42.4.081

[17] K. W. Suh, Y. J. Kwon, Infrared two-colour diagrams for AGB stars using AKARI, MSX, IRAS and near-infrared data, Mon. Not. R. Astron. Soc. 417 (2011) 3047-3060.

https://doi.org/10.1111/j.1365-966.2011.19462.x

[18] K. W. Suh, Infrared Two-Color Diagrams of AGB Stars and Planetary Nebulae Using WISE Data, J. Korean Astron. Soc. 51 (2018) 155-164. https://doi.org/10.5303/JKAS.2018.51.5.5

[19] [K. W. Suh, Infrared Properties of Asymptotic Giant Branch Stars in Our Galaxy and the Magellanic Clouds, Astrophys. J. 891 (2020) 43. https://doi.org/10.3847/1538-4357/ab6609

[20] S. L. Schnee et al., A complete look at the use of IRAS emission maps to estimate extinction and dust temperature, Astrophys. J. 634 (2005) 442. https://doi.org/10.1086/491729

[21] X. Dupac et al., Inverse temperature dependence of the dust submillimeter spectral index, A \& A. 404 1 (2003) L11-L15. https://doi.org/10.1051/0004-6361:20030575

[22] K. Young et al., Circumstellar shells resolved in IRAS survey data. II-Analysis, Astrophys. J. 409 (1993) 725-738.

https://ui.adsabs.harvard.edu/abs/1993ApJ...409..7 $\underline{25 \mathrm{Y}}$

[23] I. A. Zelko, D. P. Finkbeiner, Implications of Grain Size Distribution and Composition for the Correlation between Dust Extinction and Emissivity, Astrophys. J. 904 (2020) 38. https://doi.org/10.3847/1538-4357/abbb8d

[24] R. H. Hildebrand, The determination of cloud masses and dust characteristics from submillimetre thermal emission, Q. J. R. Astron. Soc. 24 (1983) 267.

https://ui.adsabs.harvard.edu/abs/1983QJRAS..24.. $\underline{267}$

[25] L. Sommovigo et al., Warm dust in high-z galaxies: origin and implications, Mon. Not. R. Astron. Soc. 497(2020) 956-968. https://doi.org/10.1093/mnras/staa1959

[26] E. Holmberg, On the apparent diameters and the orientation in space of extragalactic Nebulae, Meddelanden fran Lunds Astronomiska Observatorium Serie II. 117 (1946) 3-82. https://ui.adsabs.harvard. /abs/1946MeLuS.117....3H 